\title{
Zur BauchschuBfrage.
}

Von Stabsarzt d. R. Dr. L. Reichenbach, Chirurg beim Fcldlazarett.

Dic Iehre, daß im Kriege Bauchschüsse ,konservativ" zu behandeln scien, ist längst nicht mehr .,anerkanntes" Dogma und Richt. sehnur. Gesammelte Beobachtungen haben hinreichend erwiesen, da ß ,konservativ" geheilte Bauchschüsse - endgültig geheilte und wirkliche Bauchschiisse, nicht etwa Bauchwandschüsse oder Durchschïsse ohne Eingeweideverletzungen - cinen zu seltenen Glücksfall darstellen, um als richtunggebend für dic Wahl des nichtoperativen therapeutischen Verfahrens dienen zu können. Die operative Therapic hat auch für den Krieg ihr Bürgerrecht durehgesetzt. Unzweifelhaft aber bleiben die Frgebnisse der Kriegschirurgie hinter denen der Friedenschirurgic - anatomisch gleiche Verletzungen gedacht -. auf diescm Gebiete nicht unwesentlich zurück. Dic Ursachen dafür diürften hauptsächlich in der verminderten Widerstandsfähigkeit des in Kriegsstrapazen stehenden Körpers und Nervensystems liegen, anderseits auch in der Schwierigkeit, unter Feldverhältnissen allc die Forderungen zu erfüllen, die für erfolgreiche Bauchchirurgic Bedingung sind. Das Bestreben. dic operativen Resultate zu verbessern, stellt cinc Reihe von Einzclaufgaben, Kleine Bciträge aus verschiedenen Kampfgebieten erscheinen darum nicht wertlos.

Im Laufe der Sommermonate vorigen Jahres habe ich an der Westfront 21 Bauchschïsse oder Bauchverletzungen dureh Verschüt. tung behandelt. Von den mit Nierenverletzungen komplizierten sche ich ab. Sämtliche Fälle habc ich gperiert.

Das Feldlazarett war etwa $9 \mathrm{~km}$ hinter dor Front in einem Kloster eingerichtet. Die äußere Einrichtung (Operations- und Krankenräume, Beleuchtung. Behcizung) war recht gut. Das Instrumentariun war durch ïberplanmäßig gelicfertc Stücke bereichert. Operatcur, Assistenten in erforderlicher Anzahl, Operations- und Pflcgepersonal hatten sich vorziiglich aufeinander eingearbeitct. Mehrere Schwestern, eine davon als Operationsschwester, haben schr wertvolle und ganz 
ansgezciehnete Arbeit geleistet. Die Operationen konnten sich aus allen diesen Gründen in der so überaus wiehtigen hemmungslosen Weise abwickeln. Vorteilhaft war es, daB fast stets derselbe Kollege narkotisierte. Es wurde sowohl Aether als auch Chloroform angewendet, weehselnd je nach Beschaffenheit der Narkose und des Patienten. Bezïglieh der operativen Teehnik seheint mir nur erwähnenswert, daB stets reiehliehe warme Koehsalzlösung zur Warmhaltung der ausyepackten Därme und zur Spülung der Bauchhöhle verwendet wurde. Der Bauch wurde meist völlig gesehlossen. Zur Zeitersparnis wurde in der Regel die Bauehwunde mit durehgreifenden Seidennähten genäht.

Die Transportverbältnisse waren günstig. Außer dem Automobil wurden Motorboote auf Wasserwegen benutzt. Die Verwundeten gelangten naeh frühestens anderthalb Stunden ins Lazarett, meist aber wesentlich später (Bergungsschwierigkeiten).

Obwohl der Transport sehr sehonend war, kamen die Bauch. verletzten meist in sehwer mitgenommenem Zustande an, mit kleinem, sehlechtem Pula, Blässe, Prostration. Der Verwundete wurde zunäehst in ein warmes Bett gelcgt, erhiclt sof ort Kochsalzinfusion mitAdrenalin oder Digalen, auch Koffein und Kampfer, und hatte Ruhe. Während clieser Zeit wurde er kontrolliert, die Diagnose, falls sie bei der Aufnahme nicht ganz sieher zu stellen war, ausgearbeitet, lie Entseheidung getroffen und der Zeitpunkt der Operation gewählt. Meist klangen in wenigen (2-5) Stunden die Transportnachwirkungen und der Shoek soweit ab, da $B$ der Verwundete in erheblich besserem Allgemeinzustande zur Operation kam, als bei sofortigem Eingriff. Bei Zeiehen wachsender Blutung wurde natïrlich besehleunigt operiert.

I) a - eeteris paribus - die Prognose der Operation um so besser ist, je weniger die mit der Verwundung einsetzenden pathologisehen Vorgänge fortgesehritten sind, und $d a B$ auf frühzeitiges Operieren hinzuwirken ist, ist zweifellos. Diese Frühzeitigkeit muB aber mehr durch friihzeitige Einlieferung des Verwundeten erreicht werden, als dureh ciliges Nachfolgenlassen der Operation. Der frühzeitige Transport, der ja clureh weg grundsätzlieh ganz besonders fïr Bauch verletzte uiblich ist, beugt der Peritonitisentwieklung am sichersten vor. Ob in Fällen, wo baldige Bergung des Verwundeten und Abtransport eben nicht möglich ist, der Druekverband auf den Baueh für die Fahrt den Sehaden mindern oder verhüten kann, wäre zu prüfen. Natürlich kann auch während der Ruhe im Lazarett Kotaustritt beginnen oder weiter erfolgen, aber diese Gefahr wird, wie mir seheint, rcichlich aufgewogen clureh den in der Besserung des Allgemeinzustandes liegenden Vorteil.

Die Indikation zur Opcration erachtete ich stets als gegeben, wenn eine intraabdominale Verletzung diagnostiziert werden mußte und der Allgemeinzustand einen Eingriff nicht unbedingt verbot. Bauchdeckenspannung fehlte in keinem Falle. Wo sie auf anderen Ursachen beruhte, z. B. Thoraxverletzung, ließ sich während der Ruhezeit (lureh wiederholte Untersuehung die Beurteilung des Falles siehern. In jedem meiner Fälle fand die Laparotomie dureh den Operationsbefund ihre vollste Rechtfertigung. Sehwindet in angemessener Beobachtungszeit der Verdacht im Zweifelsfalle nicht, so halte ich die Probelaparotomie für angezeigt, wenn ibr Risiko nicht größer ist als normal. Durch die Anzahl der seit der Verletzung verflossenen Stunden lieB ieh mieh nieht leiten und konnte die Erfahrung machen, da $B$ aueh jenseits der ersten acht Stunden dureh Operation glückliche Ausgänge crzielt wurden in Fällen, die sonst zugrundegegangen wären.

Das Feldlazarett ist meines Erachtens diejenige Formation, die berufen ist, das Sehicksal der Bauchverletzten zu gestalten, und hat sich ausgezeiehnet bewährt in seiner Zusammensetzung und mit den ihm gegebenen Ausgestaltungsmöglichkeiten. Es kann an jeden geeigneten Ort genügend nahe vorgesehoben werden, um keinen übermäßig langen Transport zu bedingen, und kann anderseits. das für crfolgreiche chirurgische Tätigkeit Erforderliche haben. Einzelne besonders ausgestaltete und gecignete Feldlazarette in der Hauptsache für Bauchverletzte zu bestimmen, scheint mir zweekmäBig.

Im Gegensatz zu dem auch heute noch vielfach geäuBerten Pessimismus bezüglich der Behandlung der Bauehverletzungen möehte ich sagen, daB die Bauchehirurgie im Feldlazarett eines der relativ erfreuliehsten und dankbarsten Kapitel der Kricgschirurgie ist. Wenn von den nichtoperierten Bauchsehüssen sehätzungsweise etwa 90\% sterben, von den operierten aber 30 und mebr Prozent am Leben erhalten werden und meist wieder vollwertig arbeitsfähige Mensehen zu werden versprechen, so ist ein ganz wesentlicher Fortsehritt nicht zu verkennen, der - zunäehst einmal - befriedigend ist.

Da wegen Raummangels die Anführung der einzelnen Fälle untunlich, will ieh kurz bemerken, daB dureh GewehrgesehoB verwundet wurden 9, wovon 3 heilten, 6 starben; dureh Artilleriegescho $B$ wurden verwundet 10 , Heilungen 6 , Todesfälle 4 ; versehüttet wurden 2 , beide heilten. Die Versehütteten hatten DarmzerreiBungen mit Blutung und Kotaustritt in die Bauehhöhle. Bei den 10 Todesfällen war 3 mal die Todesursache Pncumonic bei einwandfreiem Verlauf der Bauchverletzungen, $1 \mathrm{mal}$ Peritonitis von einem infizierten retro- peritonealen Hämatom am 23. Tage, $1 \mathrm{mal}$ Sepsis von einer Gas. phlegmone des Obersehenkels, $5 \mathrm{mal}$ die Sehwere der Verletzung bzw. der Shoek, nämlieh Komplikation der Darmverletzungen mit Milzzerreißung, Leberverletzung, offenem Pneumothorax oder große Ausdehnung der Darmverletzungen. Der Tol erfolgte bei den letzteren Fällen $1_{1 / 2}^{1 / 36}$ Stunden nach der Operation, die sich zum Teil auf das Notdürftigste beschränkte (Vorlagerung).

Bei kleinen Zahlen ist der Prozentsatz natürlich labil. Da es sich aber hier um nahezu völlig ungesiebtes Material handelt, wie es dio Kampffront ergibt und die vorlerste Lazarettstelle bckommt, so erseheint es von Interesse, zu erwähnen, da 3 die Heilungen $52,4 \%$ der Fälle ausmachen. 